\title{
TAGUNG
}

\section{Jahre Integrationsforschung: Rückblick und Perspektiven}

\author{
Institut für Europäische Politik*
}

50 Jahre Institut für Europäische Politik: 50 Jahre ,Integrationsverantwortung “

Seit 1959 ist das Institut für Europäische Politik (IEP) als gemeinnützige Organisation auf dem Gebiet der europäischen Integration tätig. Damit hat das IEP an der Schnittstelle von Wissenschaft, Politik, Verwaltung und politischer Bildung den Prozess der europäischen Einigung seit den Anfängen begleitet. Anlässlich des 50-jährigen Bestehens des IEP fand im Oktober 2009 die jährliche Expertenkonferenz des IEP in Zusammenarbeit mit seinem Wissenschaftlichen Direktorium statt. Der Vorsitzende des Wissenschaftlichen Direktoriums, Michael Kreile, und der Direktor des IEP, Mathias Jopp, unterstrichen in ihrer Einführung, dass es dem IEP sehr gerecht werde, die 50-Jahr-Feier auf wissenschaftliche Art mit einer Konferenz zu begehen. Auf der interdisziplinären Konferenz sprachen Politik-, Rechts- und Wirtschaftswissenschaftler sowie Vertreter der Bundesregierung und der europäischen Institutionen zu aktuellen europäischen Themen und debattierten zusammen mit den Teilnehmerinnen und Teilnehmern rückblickend und vorausschauend Fragen der europäischen Integrationsforschung. Ziel der Konferenz war es dabei, eine Zwischenbilanz der Integrationsforschung $\mathrm{zu}$ ziehen (siehe Programmübersicht).

$\mathrm{Zu}$ Beginn der Tagung übermittelte Michael Clauß die Glückwünsche des Auswärtigen Amtes zum 50-jährigen Bestehen des IEP. Auch Staatsminister Günter Gloser würdigte in einer abendlichen Festansprache die Arbeit des IEP. Dabei betonte er dessen Rolle als

\section{Jahre Institut für Europäische Politik - 50 Jahre Integrationsforschung}

Expertentagung in Zusammenarbeit mit dem Wissenschaftlichen Direktorium anlässlich des 50-jährigen Bestehens des IEP

Mit freundlicher Unterstützung der Fritz Thyssen Stiftung und der Vertretung der Freien und Hansestadt Hamburg beim Bund

Berlin, 1./2. Oktober 2009

\section{Begrüßung und Eröffnung}

Prof. Dr. Michael KREILE, Vorsitzender des Wissenschaftlichen Direktoriums des IEP; Humboldt-Universität zu Berlin

Prof. Dr. Mathias JOPP, Direktor, Institut für Europäische Politik (IEP), Berlin

\section{Grußwort}

Michael CLAUß, Ministerialdirigent, Stellv. Leiter der Europaabteilung, Auswärtiges Amt, Berlin

Eröffnungsvortrag: 50 Jahre Institut für Europäische Politik - 50 Jahre Integrationsforschung

Prof. Dr. Heinrich SCHNEIDER, Ehrenvorsitzender des Wissenschaftlichen Direktoriums des IEP; Universität Wien

\section{Kommentare}

Prof. Dr. Rudolf HRBEK, Vizepräsident der Versammlung der Kuratoren des IEP; Universität Tübingen

Arno KRAUSE, Ehrenvorsitzender des Kuratoriums, ASKO EUROPA-STIFTUNG, Saarbrücken

\section{Rapporteurin}

Tanja LEPPIK-BORK, M.A., M.E.S., Wissenschaftliche Mitarbeiterin, IEP, Berlin

* Verfasst von Anne Bercio, M.E.S.; Dr. des. Katrin Böttger; Gesa-Stefanie Brincker, M.E.S.; Dipl. Frank. Wiss. Mariella Falkenhain; Dipl. Pol. Severin Fischer; Tanja Leppik-Bork, M.A., M.E.S.; Dipl. Pol. Dominic Maugeais; Wissenschaftliche Mitarbeiterinnen und Mitarbeiter, Institut für Europäische Politik, Berlin. 
Mittler zwischen Wissenschaft, Politik und Öffentlichkeit. Für die Verbindung von Wissenschaft und Politik sei gerade die langjährige Zusammenarbeit zwischen dem IEP und dem Auswärtigen Amt ein sehr gutes Beispiel. Als einige der derzeit anstehenden groBen europäischen Themen skizzierten die beiden Vertreter des Auswärtigen Amtes die mögliche Zukunft und Umsetzung des Vertrages von Lissabon, den Beginn einer neuen Phase deutscher Europapolitik, die Wirtschafts- und Finanzkrise, den Klimaschutz, die EU-Außenpolitik und die EU-Erweiterung.

Auch Heinrich Schneider honorierte in seinem Eröffnungsvortrag die Arbeit des Instituts: vor 50 Jahren als Bildungswerk durch die Europa-Union gegründet, begleitet das IEP seither die europäische Integration. Schneider hob hervor, dass das IEP nun die „Prioritäten seines Wirkens neu definieren“ müsse, um sich weiterhin den Herausforderungen der Gegenwart und Zukunft zu stellen und seiner eigenen „Integrationsverantwortung" gerecht zu werden. Als Wunsch äußerte er dabei ein ,Mehr' an Fantasie in der Europaforschung. Mit Blick auf die zukünftige Arbeit des Instituts forderte Arno Krause in seinem Kommentar zudem eine Stärkung der Beziehungen zur Zivilgesellschaft. Rudolf Hrbek unterstrich die Leistung des IEP als Plattform zwischen Wissenschaft und Praxis sowie zwischen den Disziplinen.

Lehren aus der Vergangenheit für die Integrationsforschung von Morgen

Das erste Panel debattierte unter dem Vorsitz von Wolfgang Wessels den Wandel der Europaforschung im Laufe der Zeit. Dabei warfen die Referenten einen Blick auf die vergangenen Jahrzehnte der Integrationsforschung und entwickelten daraus Ansatzpunkte für deren Weiterentwicklung in den kommenden Jahren.

Für Gianni Bonvicini, der die Europaforschung aus der Perspektive eines italienischen Think Tanks beleuchtete, spielt die Politikfeld-
Lessons of the past - for the research of the future

Einführung und Vorsitz

Prof. Dr. Wolfgang WESSELS, Universität zu Köln

\section{Panelstatements}

A look from the think tanks

Prof. Dr. Gianni BONVICINI, Executive Vice President, Istituto Affari Internazionali, Rom

Beiträge aus dem deutschen Wissenschaftsdiskurs Prof. Dr. Dr. h.c. Beate KOHLER-KOCH, Universität Mannheim

Contributions from the Anglo-Saxon world Prof. Dr. Simon BULMER, Universität Sheffield

\section{Rapporteur}

Dipl. Pol. Severin FISCHER, Wissenschaftlicher Mitarbeiter, IEP, Berlin

Europarecht: Vom Ordoliberalismus zum Konstitutionalisierungsprogramm?

Einführung und Vorsitz

Prof. Dr. Dres. h.c. Peter-Christian MÜLLERGRAFF, Universität Heidelberg

\section{Panelstatements}

Prof. Dr. Roland BIEBER, Universität Lausanne Prof. Dr. Ulrich HUFELD, Helmut-SchmidtUniversität Hamburg

\section{Rapporteurin}

Dipl. Frank. Wiss. Mariella FALKENHAIN, Wissenschaftliche Mitarbeiterin, IEP, Berlin

\section{Festansprache}

Günter GLOSER, MdB, Staatsminister für Europa, Auswärtiges Amt, Berlin

Wirtschaftswissenschaft: Die Wirtschafts- und Währungsunion als europäische Antwort auf internationale Währungs- und Finanzkrisen Vorsitz

Prof. Dr. Rolf CAESAR, Universität Hohenheim, Stuttgart

\section{Einführung}

Prof. Dr. Ansgar BELKE, Universität DuisburgEssen

\section{Panelstatements}

Prof. Dr. Wim KÖSTERS, Ruhr-Universität Bochum

Prof. Dr. András INOTAI, Institut für Weltwirtschaft, Ungarische Akademie der Wissenschaften, Budapest

Prof. Dr. Klaus GRETSCHMANN, Generaldirektor, Generalsekretariat des Rates der Europäischen Union, Brüssel 
orientierung bei der Arbeit von unabhängigen Forschungseinrichtungen eine zunehmend wichtige Rolle. Dabei liege der strategische Vorteil von Think Tanks insbesondere in ihrer Autonomie, die es ihnen ermögliche, gleichzeitig Regierungsferne wie Netzwerkfähigkeit im europäischen Umfeld zu sichern. Mit Blick auf seine eigenen Forschungsfelder, die AuBen-, Sicherheits- und Verteidigungspolitik der Europäischen Union, betonte Bonvicini, dass sich die Qualität unabhängiger Forschungsarbeit in den vergangenen Jahren verbessert habe. Durch die Beschaffenheit der Europäischen Union als sui generis-System und ihren ständigen Umbau sei der kreativanalytische Blick, insbesondere von Nachwuchswissenschaftlern in regierungsunabhängigen Institutionen, auch in Zukunft von groBer Bedeutung.

Einen anderen Schwerpunkt setzte Beate Kohler-Koch in ihrem Vortrag über den Wandel der Integrationsforschung, indem sie vier übergeordnete Trends der Forschungsarbeit skizzierte. Die Europaforschung habe sich, so Kohler-Koch, in den vergangenen Jahren stärker auf die Europäisierungsdebatte eingelassen und sich von der reinen Integrationsforschung gelöst. Gleichzeitig seien durch die parallelen Prozesse der Vertiefung und Ausdifferenzierung vergleichende Forschungsansätze bedeutungsvoller geworden. Der Governance-Ansatz im Sinne eines ,Regierens ohne Regierung" habe an Einfluss gewonnen und eine Rückbesinnung auf normative Fragen und die Abkehr von zwischenzeitlich stark positivistisch geprägten Debatten lasse sich erkennen. Nach dieser Bestandsaufnahme widmete sich Kohler-Koch den Defiziten der Europaforschung und den gegenwärtigen Problemen des Integrationsprozesses. Dabei betonte sie insbesondere die Heterogenität der Forschung innerhalb der Europäischen Union, die Problematik des Mangels europäischer Öffentlichkeit und die Defizite des europäischen Parlamentarismus. Daraus resultiere ein beständiges Legitimationsproblem, zu dessen Bewältigung in den kommenden Jah-

\section{Rapporteure}

Anne BERCIO, M.E.S., Wissenschaftliche Mitarbeiterin, IEP, Berlin

Dipl. Pol. Dominic MAUGEAIS, Wissenschaftlicher Mitarbeiter, IEP, Berlin

Internationale Beziehungen: Durch Expansion zur Weltmacht? Erweiterungsdynamik und EU-Außenpolitik

Einführung und Vorsitz

Prof. Dr. Michael KREILE, Vorsitzender des Wissenschaftlichen Direktoriums des IEP; Humboldt-Universität zu Berlin

\section{Panelstatements}

Dr. Elfriede REGELSBERGER, Stellv. Direktorin, IEP, Berlin

Dr. Barbara LIPPERT, Forschungsdirektorin, Stiftung Wissenschaft und Politik, Berlin Dr. Antonio MISSIROLI, Director of Studies, European Policy Centre, Brüssel

\section{Rapporteurin}

Dr. des. Katrin BÖTTGER, Wissenschaftliche Mitarbeiterin, IEP, Berlin

Integrationsforschung auf dem Weg zu neuen Grenzen

\section{Einführung und Vorsitz}

Prof. Dr. Tanja BÖRZEL, Freie Universität Berlin

\section{Panelstatements}

Die EU-Forschung zwischen Policy-Orientierung und Provinzialismus

Prof. Dr. Markus JACHTENFUCHS, Hertie School of Governance, Berlin

Der quantitative Turn in der EU-Forschung Prof. Dr. Annette Elisabeth TÖLLER, FernUniversität Hagen

Der Governance-Turn in der EU-Forschung Juniorprof. Dr. Carina SPRUNGK, Freie Universität Berlin

\section{Rapporteurin}

Gesa-Stefanie BRINCKER, M.E.S., Wissenschaftliche Mitarbeiterin, IEP, Berlin

\section{Schlussfolgerungen}

Prof. Dr. Michael KREILE, Vorsitzender des Wissenschaftlichen Direktoriums des IEP; Humboldt-Universität zu Berlin

Prof. Dr. Mathias JOPP, Direktor, IEP, Berlin

ren und Jahrzehnten auch die Integrationsforschung ihren Beitrag leisten könne. 
Abschließend fasste Simon Bulmer den angelsächsischen Forschungsstand in den Integrationswissenschaften zusammen und skizzierte übergreifende Trendlinien. Dabei fiel insbesondere der Gegensatz zwischen US-amerikanischer ,grand theory " und britischer Empirie auf. Diese Unterschiede seien in den vergangenen Jahrzehnten jedoch zunehmend aufgelöst und rejustiert worden. Insgesamt sei eine vermehrte Verwendung analytischer Instrumente in der Europaforschung zu beobachten gewesen, die den übergeordneten Theorieschulen zunehmend den Rang abliefen. Bulmer schloss mit einem Plädoyer für mehr Theoriebezug in der Europaforschung.

\section{Leitbilder europarechtlicher Integrationsfor- schung: Kontinuität und Wandel}

Im Vordergrund des nächsten Panels stand die Skizzierung und Bewertung von Leitbildern der rechtswissenschaftlichen Integrationsforschung.

Der Vorsitzende des Panels Peter-Christian Müller-Graff betonte einleitend, dass sich die Narrative des Europarechts im Laufe der europäischen Integrationsgeschichte verändert hätten. Nachdem in den Gründungsjahren die organisationsdogmatische Erfassung einer neuartigen supranationalen Organisation im Mittelpunkt europarechtlicher Integrationsforschung stand, rückte mit der Gründung der Europäischen Wirtschaftsgemeinschaft die Untersuchung von Marktmechanismen mit neofunktionalem Antriebspotenzial in den Vordergrund. Spätestens mit der Einführung der Direktwahl des Europäischen Parlaments folgte schließlich ein drittes Analysemodell, dessen Hauptaugenmerk auf dem Verfassungscharakter des Gemeinschaftsrechts lag.

Müller-Graff erläuterte, dass die skizzierte Abfolge europarechtlicher Leitbilder jedoch von bestimmten Entwicklungen durchbrochen werde. So habe etwa das Leitbild der Konstitutionalisierung der Europäischen Union seit dem Scheitern des Verfassungsvertrages und der rhetorischen Verabschiedung des Verfas- sungstopos an Attraktivität verloren. Gleichzeitig bestehe unter juristischen Akteuren Konsens über die Verfassungsqualität der europäischen Rechtsordnung. Schließlich sei auch die ordoliberale Betrachtung der Europäischen Union noch heute ,wirkmächtig“, wie der Neuauftrieb der Frage nach der Integrationsrolle der Wirtschaftsordnung zeigt.

Einen linearen Wandel der Bilder des Europarechts stellte auch Roland Bieber infrage. Während sich die europäische Rechtsordnung unter anderem durch richterliche Rechtsfortbildung und Vertragsänderungen stetig wandele, blieben die Fragestellungen europarechtlicher Integrationsforschung, wie jene nach den Wechselwirkungen zwischen dem Recht der Union und mitgliedstaatlichen Rechtsordnungen, unverändert.

Der aktuellen Bedeutung des konstitutionalistisch-legitimierenden Ansatzes des Europarechts widmete sich Ulrich Hufeld. Er erläuterte, dass Konstitutionalisierung beginne, wenn sich Toleranzen in Rechte umwandeln. Das Europarecht vermittle bereits seit den frühen 1960er Jahren Freiheitsrechte statt Toleranzen. Der Primat der Freiheit, der durch das Prinzip des Vorrangs institutionell abgesichert ist, bedeute für den Unionsbürger eine Reduzierung von Herrschaft, so Hufeld weiter. In diesem Sinne seien die europarechtlichen Grundfreiheiten gerade in Zeiten eines zunehmenden Legitimationsdefizits als eine Legitimationsressource der Europäischen Union anzusehen.

Hufeld kritisierte, dass das Bundesverfassungsgericht in seinem Urteil zum Vertrag von Lissabon das Europa der Freiheiten nicht würdige. Grundfreiheiten und gemeinschaftliche Rechte würden vielmehr von Überlegungen zu Demokratie und Herrschaft überlagert. Auch Bieber und Müller-Graff äußerten sich kritisch zum Karlsruher Richterspruch. Bieber bezeichnete die ,provokative“ Betonung des Schutzgutes , nationale Souveränität" als rückwärtsgewandt. Weder das europäische Primärrecht noch das positive deutsche Ver- 
fassungsrecht stellten das Konzept des souveränen Nationalstaates als schützenswert dar. Müller-Graff thematisierte ebenfalls das Souveränitätskonzept des Bundesverfassungsgerichts und bemängelte dessen rein defensive Ausrichtung. So würde der Übergang von Einstimmigkeit zu qualifizierter Mehrheit lediglich als Gefahr für staatliche Souveränität dargestellt, das positive Gestaltungspotenzial von Mehrheitsentscheidungen aber nicht anerkannt.

,Rettungsanker oder Auslaufmodell - eine Situationsanalyse zur Krisenbeständigkeit der Wirtschafts- und Währungsunion

Tagespolitisch aktuell wurde die Diskussion auch im wirtschaftswissenschaftlichen Panel. Die Teilnehmer der vierten Diskussionsrunde debattierten die Rolle der Wirtschafts- und Währungsunion (WWU) bei der Überwindung tiefgreifender Finanzkrisen. Dabei gingen alle Referenten insbesondere auf die aktuelle Situation ein, welche die Akteure der WWU vor völlig neue Herausforderungen stelle. Übereinstimmend betonten die Teilnehmer des von Rolf Caesar geleiteten $\mathrm{Pa}$ nels, dass sich die WWU im Zuge der weltweiten Finanz- und Wirtschaftskrise als stabilisierender Faktor bewähren konnte.

Ansgar Belke stellte in seinem einführenden Beitrag dar, dass eine Entkoppelung von globalen Finanzkrisen aber auch zukünftig nicht gelingen werde, weshalb die wiederkehrenden „Blasen“ am internationalen Finanzmarkt auch weiterhin auf den Euroraum durchschlagen würden. Der derzeit vorhandene Liquiditätsüberschuss führe dabei über kurz oder lang auch in der Eurozone zu einer erhöhten Umlaufgeschwindigkeit des Geldes, was wiederum den Effekt steigender Rohstoffpreise nach sich ziehe. Die so charakterisierte ,globale Sparschwemme“ gelte es aufzuheben. Belke machte in seinem Vortrag allerdings deutlich, dass der Euroraum auch in der Vergangenheit und Gegenwart wegbrechende Exportmärkte nur unzureichend durch steigende Binnennachfrage ausgleichen konnte. Demzu- folge sei auch für die nahe Zukunft den Vereinigten Staaten ein größeres Wachstumspotenzial als dem Euroraum zu prognostizieren.

In Bezug auf die Geldpolitik wagte Belke eine eher verhaltene Diagnose. Fakten, die für eine Beibehaltung des US-Dollars als weltweite Leitwährung sprächen, lägen auf der Hand: Die weltweite Reservenakkumulation geschieht auf einem gleichbleibend hohen Niveau in US-Dollar. Gleichzeitig werden externe und fiskalische Defizite in den Vereinigten Staaten derzeit reduziert, während die Entwicklung im Euroraum den entgegengesetzten Trend nachzeichnet. Auch den Mangel an ,politischem Potenzial“ in Europa, an der Neuordnung der globalen Finanzordnung entscheidend mitzuwirken, kritisierte Belke. Es müsse ein Ziel der kommenden zehn Jahre sein, die politische Integration der Märkte des Euroraums über den Wirkungsrahmen der WWU hinaus bis hin zu gemeinsamen Finanzinstitutionen und einer gemeinsamen Repräsentanz in globalen Finanzinstitutionen zu führen. Dann, so Belke, würde die geldpolitisch bessere Ausgangslage der WWU im Vergleich zu den USA auch zum Tragen kommen und der Euro im Hinblick auf die internationale Leitwährung mittel- bis langfristig zum US-Dollar in Konkurrenz treten.

In seinem Panelstatement erläuterte Wim Kösters, dass die Unabhängigkeit der Europäischen Zentralbank (EZB) für eine erfolgreiche Geldpolitik im Kontext der Finanz- und Wirtschaftskrise elementar ist und bleibe. Aufkeimenden Diskussionen mit Blick auf mögliche Beschränkungen dieser absoluten Unabhängigkeit der EZB, sei mit größter Vorsicht zu begegnen. Kösters betonte, dass sich die WWU insbesondere auch deshalb in der Krise bewährt hätte, da durch den Euro währungspolitische Verwerfungen verhindert wurden. Starke innereuropäische Ungleichgewichte (Stichwort: Staatsdefizite) stellten jedoch für die Europäische Währungsunion (EWU) eine große Herausforderung dar. Der Erfolg der EWU sei deshalb keineswegs garantiert und müsse kontinuierlich erarbeitet 
werden. András Inotai kritisierte, dass allein auf Geldpolitik basierende Krisenlösungsmechanismen zu weitreichenden Problemen, wie zum Beispiel einer ausnahmslos hohen Staatsverschuldung führten, sodass möglichst zügig auch über mögliche Exit-Strategien nachgedacht werden müsse.

Als Vertreter aus der Politik äußerte sich abschließend Klaus Gretschmann erfreut über den positiven und geeinten Umgang der Europäischen Union mit der globalen Finanz- und Wirtschaftskrise. Bei näherer Betrachtung handele es sich bei den Reaktionen der Mitgliedstaaten jedoch vornehmlich um nationale und sektorale Krisenbekämpfungsinstrumente. Innerhalb der Union sei mit Konflikten zwischen den Mitgliedstaaten und der Europäischen Kommission hervorragend umgegangen worden und auch nach außen sei die Europäische Union geschlossen aufgetreten. Gretschmann betonte ebenfalls, dass es an der Zeit sei, die Aufmerksamkeit von den Krisenlösungsmechanismen auf mögliche Auswege aus der Verschuldungsproblematik zu richten.

Ob die Europäische Union in den internationalen Beziehungen eine ähnlich fortentwickelte Akteursqualität aufweist wie in der wirtschaftlichen Anti-Krisenpolitik, wurde im folgenden Panel diskutiert.

Integrationsmotor oder außenpolitischer Akteur? Die Rolle Europas in der Welt

Das Panel schrieb der Europäischen Union insgesamt eine zunehmende aber noch punktuelle Akteursqualität zu. Der Vorsitzende des Panels Michael Kreile betrachtete als wichtigsten Problembereich in diesem Zusammenhang den Zielkonflikt zwischen Handlungsfähigkeit und Erweiterung der Europäischen Union.

Elfriede Regelsberger sagte einleitend, dass der Ansatz der IEP-Forschung zur Gemeinsamen Außen- und Sicherheitspolitik (GASP) immer gewesen sei, nach der, nature of the beast` $\mathrm{zu}$ fragen. Regelsberger betonte, dass die Europäische Union weder ein außenpoliti- scher Zwerg noch eine Weltmacht sei, aber über Akteursqualität verfüge. So stellte sie bei ihrer Analyse der Instrumente und Akteure der GASP qualitative und quantitative Wachstumstrends fest, die sich sowohl auf die klassischen Instrumente (Deklarationen, Reise- und Vermittlungstätigkeit und politischer Dialog) als auch auf die neuen Instrumente (insbesondere gemeinsame Aktionen, vor allem in Form des zivilen und militärischen Krisenmanagements, und die Sonderbeauftragten) bezogen. Zudem gebe es auch eine wachsende Anzahl von Akteuren, welche die Sichtbarkeit und Kontinuität der GASP sichern helfen. Ausblickend dürfe man gespannt sein, wie sich die Rolle des Hohen Vertreters für die GASP und des Europäischen Auswärtigen Dienstes mit dem Lissabonner Vertrag ändern werde.

In ihrem Vortrag leuchtete Barbara Lippert aus, wie es zur Erweiterungsdynamik und der darauffolgenden Krise der Erweiterung gekommen ist und was daraus gelernt werden kann. Die Anziehungskraft der Europäischen Union ergebe sich aus ihrer Wirtschaftskraft und ihren Fähigkeiten, als Modernisierungsund Demokratisierungshebel zu wirken. Die Europäische Union habe dabei die Erweiterungsdynamik stets aus der Integrationsdynamik heraus erklärt und nie als ein strategisches Ziel der Machtentfaltung verstanden. Erst die EU-Osterweiterung wurde mit geopolitischen Konzepten und dem Begriff der, soft power' unterlegt.

Da die Erweiterungspolitik aber ins Stocken geraten sei, so Lippert, müsse die Europäische Union umsteuern und zwischen Erweiterung und Außenpolitik differenzieren, um eine Fehlentwicklung der Erweiterungspolitik in Richtung „Mädchen für alles“ zu verhindern. Deshalb solle die Europäische Union Beitrittsverhandlungen künftig zeitlich begrenzen und sich darüber klar werden, ob ein Land tatsächlich aufgenommen werden soll, anstatt der bisher existierenden Prozessautomatik zu folgen. Hieraus ergab sich für Lippert die spannende Frage, was es für das Rollenbild 
der Europäischen Union bedeuten könnte, wenn sie sich von der Politik der offenen Tür verabschiede.

Auch Antonio Missiroli befasste sich mit der Frage, wie die Erweiterungspolitik in das breitere Ensemble der Außenpolitiken hineinspielt. Er bezeichnete die Erweiterungspolitik als eine ,zufällige“ Außenpolitik mit anderen Mitteln, die sich auch sprachlich von anderen Politiken unterscheide, da zum Beispiel nicht von ,Expansion“ die Rede sei. Laut Missiroli steht die Erweiterungspolitik heute an einem Scheidepunkt, da sie nicht mehr ausreicht um Reformen in der Türkei oder den Staaten des Westlichen Balkans voranzutreiben und da die Konditionalität viel weniger effektiv funktioniert als das noch bei der Osterweiterung der Fall war. Vor diesem Hintergrund schätzte Missiroli die Europäische Nachbarschaftspolitik (ENP) als die ,Wachstumsindustrie " in der Wissenschaft ein: Jedoch handele es sich bei der ENP um eine unausgereifte Politik, die weder ausschließlich als Erweiterungsnoch als Außenpolitikinstrument betrachtet werden könne.

Die Integrationsforschung der Zukunft: neue theoretische und praktische Herausforderungen

Nach inhaltsreichen und in Teilen kontrovers geführten Debatten kamen die Teilnehmerinnen und Teilnehmer der zweitägigen Konferenz zu dem Schluss, dass sich die „Integrationsforschung auf dem Weg zu neuen Grenzen" befinde. So sei bemerkenswert, betonte die Leiterin des letzten Panels, Tanja Börzel, dass sich die EU-Forschung in Deutschland inzwischen als eigenes Feld etabliert habe. Mit Blick auf die Herausforderungen, mit denen die Integrationsforschung zu Beginn des 21. Jahrhunderts konfrontiert sei, bemängelte Markus Jachtenfuchs die fehlende Distanz zum Untersuchungsgegenstand und die zu starke Orientierung an der politischen Agenda. Eine zu starke Policy-Orientierung könne sich dann für die Europaforschung als gefährlich erweisen, wenn sich die
Wissenschaft der Attraktivität der zwar aktuellen doch zumeist schnelllebigen Themen der Policy-Akteure unterwerfe. Weder die Europäische Union noch ihre Agenda dürfe aufgrund ihrer Einmaligkeit als vorbehaltlos positiv betrachtet werden. Anders als vor etwa 30 Jahren bestehe kein Informationsdefizit mehr, weder die Bürger noch politische Akteure müssten von den Vorteilen europäischer Integration überzeugt werden. Stattdessen sei die Europäische Union ,politisch angekommen“. Demzufolge forderte Jachtenfuchs mehr Mut zu streitbarer EU-Forschung und eine Abkehr von unkritischer Europagläubigkeit. Carina Sprungk schloss sich dieser Argumentation an und betonte die Notwendigkeit, die sui generis-Debatte zu überwinden, die ohnehin eine intern beschränkte Sichtweise sei. Sprungk berichtete demgegenüber von der Außenwahrnehmung der europäischen Integrationsforschung, die beispielsweise in den USA vielmehr als Teilbereich internationaler Politik gewertet werde. Als Entwicklungsmöglichkeiten skizzierte die Referentin eine stärkere Verknüpfung der EUForschung mit den klassischen Themen der Politikwissenschaft. Wenn das politische System der Europäischen Union als gegeben angesehen werde, könne der Fokus der Integrationsforschung zukünftig stärker auf komparative Politikwissenschaft und Regierungslehre gelegt werden. In diesem Kontext solle der ,governance turn' in der Integrationsforschung die gegenseitige Befruchtung mit anderen Disziplinen ermöglichen. Governance-Forschung könne zudem eine Ausweitung des Blicks auf Privatakteure und Bürger bewirken sowie eine Verbindung zwischen nationaler und internationaler Ebene herstellen.

Anschließend erläuterte Annette Elisabeth Töller, dass im politikwissenschaftlichen „Mainstream“ ein qualitativer ,turn', in der Integrationsforschung dagegen ein quantitativer , turn' stattgefunden habe. Die zwei Varianten quantitativer EU-Forschung (quantifizierend-beschreibende beziehungsweise quantitativ-erklärende Studien) trügen beide 
zu einer stärkeren Überprüfbarkeit des tatsächlichen Einflusses der europäischen auf die nationale Gesetzgebung bei. Töller verneinte zwar, dass ein ,nennenswerter“ quantitativer ,turn" stattgefunden habe, unterstrich jedoch, dass statistische Erfassungen im Rahmen der EU-Forschung - beispielsweise die quantitative Zusammenstellung von Rechtsakten - eine gewisse Systematisierung und einen Erkenntnisgewinn ermöglichten. Auf diese Weise könnte Mythenbildungen, wie dem 80-Prozent-Mythos zur EU-Gesetzgebung, entgegen gewirkt werden. Aufgrund der Komplexität des EU-Systems bleibe jedoch eine mangelnde Aussagekraft in einzelnen Bereichen bestehen. Zudem könnten unterschiedliche Methoden zu diversen Ergebnissen führen. Töller erhoffte sich jedoch als zukünftiges Ergebnis des quantitativen ,turns", trotz seiner Schwächen, einen stärkeren Anschluss der Europaforschung an den Mainstream der Politikwissenschaft.

Einen abschließenden Blick auf praktische Problemlagen transnationaler EU-Forschung warf Gesa-Stefanie Brincker. Sie betonte, dass mit der Erweiterung von 2004/2007 zwar zahlreiche neue Möglichkeiten der Kooperation zwischen Wissenschaftlern aus Westund Mittelosteuropa entstanden seien. Der da- mit zutage tretenden Verschiedenheit in den einzelnen Forschungskulturen, welche die alltägliche gemeinsame Forschungsarbeit erschwerten, werde jedoch noch nicht ausreichend begegnet. So müsse im Kontext der zunehmend transnationalen Forschungsvorhaben zukünftig ein stärkerer Fokus auf die konkrete Realisierbarkeit der Projekte gelegt werden - und zwar im gegenseitigen Austausch zwischen den Forschern unterschiedlicher Herkunft. Brincker betonte, dass nur mittels gemeinsam erarbeiteter Lösungen die alltägliche Forschungskooperation über Grenzen hinweg erleichtert werden könne.

\section{Fazit}

Insgesamt machte die Konferenz einmal mehr deutlich, wie befruchtend der Austausch zwischen Wissenschaftlern verschiedener Disziplinen sowie zwischen Wissenschaft und Praxis sein kann - dies umso mehr, wenn es sich um ein so komplexes sowie sich stetig wandelndes Gebilde handelt wie die Europäische Union. Gerade angesichts neuer interner wie externer Herausforderungen an die Europäische Union bedarf es deshalb auch in der $\mathrm{Zu}$ kunft einer Weiterführung der Integrationsforschung innerhalb sowie im Austausch zwischen den Disziplinen.

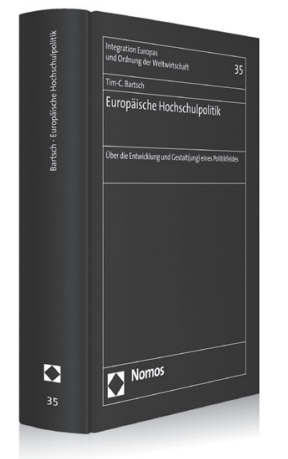

\section{Europäische Hochschulpolitik \\ Über die Entwicklung und Gestalt(ung) eines Politikfeldes \\ Von Dr. Tim-C. Bartsch}

2009, 404 S., geb., 79,-€,

ISBN 978-3-8329-4716-3

(Integration Europas und Ordnung

der Weltwirtschaft, Bd. 35)

Bitte bestellen Sie im Buchhandel oder

versandkostenfrei unter $>$ www.nomos-shop.de

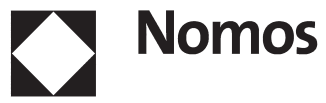

